\title{
Estrategias convergentes: Estudio de las tendencias en la incorporación de recursos interactivos y de distribución en los principales grupos de comunicación en España (2006-2008)
}

\section{Convergences strategies: Research of the trends in the incorporation of the interactive resources and distribution platforms in the main communication groups in Spain (2006-2008)}

Silvia Martínez Martínez. Universitat Oberta de Catalunya (UOC)

Recibido: 15-XI-2011 - Aceptado: 7-V-2013

Resumen:

La digitalización del mensaje ha favorecido el establecimiento de sinergias en los grupos de comunicación. Gracias al potencial y la cooperación entre medios del grupo, la propiedad puede influir en la adaptación del medio al entorno digital. En este contexto el estudio analiza la sintonía y homogeneidad en la oferta de aplicaciones interactivas y plataformas de distribución en doce grupos de comunicación españoles entre 2006 y 2008 para establecer tendencias en su evolución. Los resultados demuestran que existen distintos niveles de correspondencia si bien se registra un incremento en el número de variables que presentan todas las cabeceras del grupo.

Palabras clave:

Grupos de comunicación en España, interactividad, Web 2.0, UGC, plataformas de distribución.

Abstract:

The digitalization of the message has favored the establishment of synergies in the media groups. Due to the development of strategies for internal cooperation, the property may influence the adaptation of the media to the digital environment. In this context, the study analyzes the uniformity and homogeneity in the provision of interactive applications and distribution platforms in twelve groups of Spanish press between 2006 and 2008 to establish trends in their evolution. The results demonstrate there are different levels of correspondence although it is registered an increase in the number of variables that are present in all the diaries of the group

Keywords:

Communication groups in Spain, interactivity, Web 2.0, UGC, distribution platforms. 


\section{Los grupos de comunicación en España: entre la concentración y la convergencia}

Desde la implantación de la democracia, uno de los rasgos que ha caracterizado al sistema de medios español es la "tendencia a la concentración” (Boix y López García, 2006:169). Según Nuria Almirón (2009), este proceso implica que unas pocas compañías acaparan el negocio en términos de ingresos y alcanzan grandes cuotas de audiencia. Así, en 2010, en el sector de la prensa, Unedisa y Prisa obtienen las cifras de lectores más elevadas aunque los grupos con marcado componente regional representan un alto porcentaje sobre los datos totales (Santamaría 2011:163).

El proceso de concentración se ha visto traspasado por la convergencia, la digitalización y, con ello, la aparición de nuevos actores (Becerra y Mastrini, 2006). En Internet se desdibujan las barreras de entrada y se facilita la publicación de contenidos. Junto a los medios convencionales, irrumpen en la Red nuevos sujetos y se enriquece el panorama mediático. Pero, en contraste con esta apertura, siguen dominando el entorno comunicativo unos pocos medios que pertenecen a los grandes grupos o a "las principales empresas de telecomunicaciones" (Boix y López García, 2006: 167). Este protagonismo transciende a las cabeceras puesto que los "grupos editoriales a los que pertenecen están ocupando el Top 10 de las primeras compañías de Internet en España" (IAB, 2010: 12).

En este contexto la marca se presenta como un componente con más relevancia que el propio soporte (Salaverría, 2010:29). Se trata de un elemento clave en la estrategia mediática para atraer la atención de la audiencia. Junto a ella, los grupos se ven favorecidos por los movimientos de integración o de convergencia multimedia entendida como "la búsqueda de productos informativos cualitativamente mejores a través de la cooperación entre medios” (Salaverría, 2003) y que se beneficia del desarrollo de las sinergias entre las distintas unidades del grupo. Estos procesos se completan con la puesta en marcha de cambios estructurales y aparecen unidades organizadas que coordinan esfuerzos para obtener mayores rentabilidades. Como ejemplos de este proceso Nereida Cea (2009:3) menciona, entre otros, la creación de Mundinteractivos o Unidad Editorial Internet, Prisacom o Zeta Digital.

Fernando Ramos Simón (1996) señalaba que una de las características de los grupos de comunicación multimedia reside en la búsqueda de beneficios a partir de la aplicación de las sinergias y la cooperación interna. Con la digitalización del mensaje, adaptarse a las características de la comunicación en Red aporta valor añadido al producto informativo. La adopción de recursos interactivos y de la Web 2.0, la incorporación de opciones de UGC y nuevas vías de distribución son aspectos que tienen consecuencias en las estrategias asumidas por las compañías mediáticas. La implementación de recursos en los medios online ha ido registrando varios estadios de desarrollo y se ha visto influenciada por distintos factores de modo que, aunque se puede hablar de evolución e innovación en los medios de comunicación, estos "en muchas ocasiones han mostrado tendencias y estrategias dependientes de variables económicas y empresariales” (Díaz Noci, 2010). 


\section{Relación entre pertenencia y dimensión del medio y la oferta de recursos interactivos y plataformas de distribución}

La relación entre oferta de aplicaciones interactivas en el entorno digital y la dimensión del medio ha sido observada tempranamente en diversos estudios. La investigación longitudinal realizada por Greer y Mesing (2006) sobre 83 cabeceras estadounidenses entre 1997 y 2003 demuestra no sólo la evolución positiva de recursos sino que, además, los medios con datos elevados o medianos de difusión presentan mayores cuotas de sofisticación. También en EEUU, Schultz (1999), al analizar las opciones interactivas en 100 diarios, observa que los periódicos más pequeños son los que presentan resultados más bajos. Asimismo, señala que la estructura de la propiedad no era un buen factor de predicción de la implementación de las variables estudiadas aunque indica que la media obtenida por los medios pertenecientes a alguna cadena era superior a la de los periódicos independientes. Una investigación de los diarios digitales de referencia en España, Argentina y México permite a Albornoz (2007) concluir que factores exógenos y endógenos influyen en las características que presentan los medios en Internet. Entre estos últimos menciona el prestigio del diario pero también su pertenencia a importantes grupos de comunicación. Conclusiones similares se registran en análisis centrados en el contexto español donde, en un estudio desarrollado en 2003, se afirma que los diarios de primera línea "se caracterizan por una difusión alta, la pertenencia a grupos multimedia (en particular destaca el grupo Vocento, antes Correo Prensa Española) y por el equilibrio en las puntuaciones obtenidas en las variables relativas a recursos informativos y valor de portal" (García de Torres et al, 2004a:11).

Martínez Méndez y López Carreño (2005:58), en un estudio prospectivo sobre páginas Web españolas, concluyen que los "niveles de desarrollo se ven tan influenciados por la potencia del grupo que respalda al portal como por la calidad de su diseño". La presencia de perfiles diversos al comparar los grupos queda plasmada en investigaciones sobre el desarrollo del portal en las que sobresale Prisa (López Carreño, 2004) o sobre las posibilidades interactivas y multimedia con una presencia destacada de Correo Prensa Española/Vocento (García de Torres et al. 2004b). Martínez Martínez (2007), al analizar el perfil específico de la prensa especializada, también registra la relación entre posicionamiento del diario y oferta de herramientas y observa que Prisa presenta mayor número de aplicaciones interactivas y de plataformas de distribución.

La diversidad de aprovechamiento de las posibilidades que brinda Internet en función del grupo de comunicación contrasta con la homogeneidad interna registrada en otros trabajos. Así, sobre los 31 ítems analizados para determinar los contenidos de servicios, el estudio realizado por el grupo de investigación de la Universidad CEU Cardenal Herrera (García de Torres et al. 2004c) muestra mayor correspondencia en la presencia de herramientas analizadas en las cabeceras de Vocento. En un análisis sobre noticias de portada, donde si bien Vocento muestra un perfil más completo, Joly destaca en uniformidad y se deriva la existencia de "una tendencia a la homogeneidad, en términos cuantitativos y cualitativos, expresión de sinergias de grupo” (García de Torres et al., 2006:353). En un estudio posterior realizado por García de Torres, 
Rodríguez Martínez y Martínez Martínez (2010) se analiza la correspondencia entre la oferta de recursos Web 2.0 y la presencia de herramientas de control en los principales grupos de comunicación españoles. La investigación concluye que existe una sintonía media/alta por la presencia o ausencia de recursos.

\section{Metodología}

El objetivo de la presente investigación es determinar los patrones de correspondencia en la oferta de recursos interactivos y de distribución entre los diarios de los principales grupos de comunicación españoles. Ello permitirá apuntar la presencia y evolución de políticas conjuntas o estrategias de grupo en la incorporación de este tipo de herramientas.

Estudios previos que han demostrado la influencia de la posición en el mercado, tanto en términos de difusión como de propiedad, sirven de base para establecer algunas de las hipótesis del estudio. Así, (H1) se espera registrar unos niveles de incorporación de herramientas altos entre los grupos de comunicación estudiados con una evolución positiva en el periodo y la presencia destacada de ellas en diarios líderes (García de Torres et al. 2008). No obstante, (H2) se espera obtener un nivel de sintonía media o alta aunque con distintos grados de desarrollo de modo que existan algunos grupos más destacados coincidiendo con los resultados obtenidos por el estudio de García de Torres, Rodríguez Martínez y Martínez Martínez (2010). Por herramientas, (H3) se espera observar en los grupos de comunicación una mayor apuesta por implementar en sus diarios recursos como el weblog, la opción de comentar los contenidos y, entre las opciones de distribución, la posibilidad de sindicar los contenidos (RSS), ello conforme a la popularización de estas herramientas registrada en estudios previos (Domingo et al, 2007, 2008)

Para dar respuesta al objetivo de la investigación y comprobar las hipótesis se parte de los resultados del análisis realizado en los meses de junio y julio de 2006, 2007 y 2008 y que fueron presentados en la tesis doctoral La Interactividad y las plataformas de distribución de los medios online de referencia. Tendencias en la configuración del nuevo entorno informativo digital español ${ }^{1}$. En este artículo de manera específica se presentan no solo las tendencias en la incorporación de las herramientas sino que se profundiza en las pautas de sintonía y homogeneidad entre las distintas cabeceras para definir las estrategias de coordinación interna de los grupos. La muestra, que fue establecida a partir de la relación de diarios publicados en el mes de mayo de 2006, 2007 y 2008 por la OJD y la OJD Interactiva (incluyendo los medios generalistas y especializados, de pago y gratuitos), se compone de las cabeceras con presencia en Internet de los principales grupos de comunicación. Para fijar la selección de compañías se parte de los datos que figuran en la revista Noticias de la Comunicación (2007:56-58; 2008:52-54) y se contacta con algunas cabeceras para confirmar la propiedad. Asimismo, para poder

1 Tesis doctoral defendida el 4 de abril de 2011 por Silvia Martínez bajo la dirección de la doctora Elvira García de Torres. En su desarrollo contó con una beca de formación de personal universitario del Ministerio de Educación (BFPU AP2005-4591). Además la investigación se encontraba enmarcada en un proyecto financiado por el Ministerio de Ciencia e Innovación (SEJ200614595SOCI). 
realizar la comparativa que permita marcar la evolución y las tendencias en sintonía y homogeneidad interna se establecen dos requisitos adicionales: que el grupo presente más de un periódico en aquellos listados y que tenga vigencia durante los tres años analizados. Por consiguiente en este artículo se estudian 12 grupos de comunicación (El Progreso, Editorial Prensa Ibérica, Godó, Grupo Heraldo, Joly, La Región, Prisa, Promecal, Serra, Unidad Editorial/Unedisa, Vocento y Zeta) y un total 82 cabeceras: 68 de ellas son analizadas en 2006, 70 en 2007 y 75 en 2008.

Tabla 1. Selección de la muestra: grupos de comunicación y diarios (2006-2008)

\begin{tabular}{|c|c|c|c|}
\hline Grupo & 2006 & 2007 & 2008 \\
\hline El Progreso (PG) & $\begin{array}{l}\text { Diario de Pontevedra } \\
\text { El Progreso }\end{array}$ & $\begin{array}{l}\text { Diario de Pontevedra } \\
\text { El Progreso } \\
\text { LV de Luns a Venres }\end{array}$ & $\begin{array}{l}\text { Diario de Pontevedra } \\
\text { El Progreso } \\
\text { LV de Luns a Venres }\end{array}$ \\
\hline $\begin{array}{l}\text { Editorial Prensa Ibérica } \\
(\mathrm{EPI})\end{array}$ & $\begin{array}{l}\text { Diari de Girona } \\
\text { Diario de Ibiza } \\
\text { Diario de Mallorca } \\
\text { Faro de Vigo } \\
\text { Información } \\
\text { La Nueva España } \\
\text { La Opinión/ El Correo de Za- } \\
\text { mora } \\
\text { La Opinión de A Coruña } \\
\text { La Opinión de Granada } \\
\text { La Opinión de Málaga } \\
\text { La Opinión de Murcia } \\
\text { La Opinión de Tenerife } \\
\text { La Provincia } \\
\text { Levante-EMV } \\
\text { Regió } 7 \\
\text { Súper Deporte }\end{array}$ & $\begin{array}{l}\text { Diari de Girona } \\
\text { Diario de Ibiza } \\
\text { Diario de Mallorca } \\
\text { Faro de Vigo } \\
\text { Información } \\
\text { La Nueva España } \\
\text { La Opinión/El Correo de Za- } \\
\text { mora } \\
\text { La Opinión A Coruña } \\
\text { La Opinión de Granada } \\
\text { La Opinión de Málaga } \\
\text { La Opinión de Murcia } \\
\text { La Opinión de Tenerife } \\
\text { La Provincia } \\
\text { Levante-EMV } \\
\text { Regió } 7 \\
\text { Súper Deporte }\end{array}$ & $\begin{array}{l}\text { Diari de Girona } \\
\text { Diario de Ibiza } \\
\text { Diario de Mallorca } \\
\text { Faro de Vigo } \\
\text { Información } \\
\text { La Nueva España } \\
\text { La Opinión/ El Correo de Za- } \\
\text { mora } \\
\text { La Opinión A Coruña } \\
\text { La Opinión de Granada } \\
\text { La Opinión de Málaga } \\
\text { La Opinión de Murcia } \\
\text { La Opinión de Tenerife } \\
\text { La Provincia } \\
\text { Levante-EMV } \\
\text { Regió } 7 \\
\text { Súper Deporte }\end{array}$ \\
\hline Godó (GD) & $\begin{array}{l}\text { La Vanguardia } \\
\text { Mundo Deportivo }\end{array}$ & $\begin{array}{l}\text { La Vanguardia } \\
\text { Mundo Deportivo }\end{array}$ & $\begin{array}{l}\text { La Vanguardia } \\
\text { Mundo Deportivo }\end{array}$ \\
\hline
\end{tabular}

2 Aparecen entre los medios auditados por la OJD pero no ofrece datos de difusión en 2007. 
Estrategias convergentes: Estudio de las tendencias en la incorporación de recursos interactivos...

\begin{tabular}{|c|c|c|c|}
\hline Grupo & 2006 & 2007 & 2008 \\
\hline Grupo Heraldo $(\mathrm{H})$ & $\begin{array}{l}\text { Heraldo de Aragón } \\
\text { Heraldo de Soria }\end{array}$ & $\begin{array}{l}\text { Heraldo de Aragón } \\
\text { Heraldo de Soria }\end{array}$ & $\begin{array}{l}\text { Heraldo de Aragón } \\
\text { Heraldo de Soria }\end{array}$ \\
\hline Joly (J) & $\begin{array}{l}\text { Diario de Cádiz } \\
\text { Diario de Jerez } \\
\text { Diario de Sevilla } \\
\text { El Día de Córdoba } \\
\text { Europa Sur } \\
\text { Granada Hoy } \\
\text { Huelva Información } \\
\text { Málaga Hoy }\end{array}$ & $\begin{array}{l}\text { Diario de Cádiz } \\
\text { Diario de Jerez } \\
\text { Diario de Sevilla } \\
\text { El Día de Córdoba } \\
\text { Europa Sur } \\
\text { Granada Hoy } \\
\text { Huelva Información } \\
\text { Málaga Hoy }\end{array}$ & $\begin{array}{l}\text { Diario de Cádiz } \\
\text { Diario de Jerez } \\
\text { Diario de Sevilla } \\
\text { El Día de Córdoba } \\
\text { Europa Sur } \\
\text { Granada Hoy } \\
\text { Huelva Información } \\
\text { Málaga Hoy } \\
\text { Almería Actualidad }^{3}\end{array}$ \\
\hline La Región (LR) & $\begin{array}{l}\text { Atlántico diario } \\
\text { La Región }\end{array}$ & $\begin{array}{l}\text { Atlántico diario } \\
\text { La Región }\end{array}$ & $\begin{array}{l}\text { Atlántico diario } \\
\text { La Región }\end{array}$ \\
\hline Prisa $(\mathrm{P})$ & $\begin{array}{l}\text { As } \\
\text { Cinco Días } \\
\text { El Correo de Andalucía } \\
\text { El País } \\
\text { Jaén } \\
\text { Odiel Información }\end{array}$ & $\begin{array}{l}\text { As } \\
\text { Cinco Días } \\
\text { El País }\end{array}$ & $\begin{array}{l}\text { As } \\
\text { Cinco Días } \\
\text { El País }\end{array}$ \\
\hline Promecal (PR) & $\begin{array}{l}\text { Diario de Ávila } \\
\text { Diario de Burgos } \\
\text { Diario Palentino } \\
\text { El Adelantado de Segovia } \\
\text { La Tribuna de Ciudad Real }\end{array}$ & $\begin{array}{l}\text { Diario de Ávila } \\
\text { Diario de Burgos } \\
\text { Diario Palentino } \\
\text { El Adelantado de Segovia } \\
\text { La Tribuna de Ciudad Real }\end{array}$ & $\begin{array}{l}\text { Diario de Ávila } \\
\text { Diario de Burgos } \\
\text { Diario Palentino } \\
\text { El Adelantado de Segovia } \\
\text { La Tribuna de Ciudad Real } \\
\text { La Tribuna de Albacete } \\
\text { La Tribuna de Cuenca } \\
\text { La Tribuna de Guadalajara } \\
\text { La Tribuna de Talavera } \\
\text { La Tribuna de Toledo }\end{array}$ \\
\hline
\end{tabular}

\footnotetext{
Aparece entre los medios auditados por la OJD pero no ofrece datos de difusión en 2008.
} 


\begin{tabular}{|l|l|l|l|}
\hline \multicolumn{1}{|c|}{ Grupo } & \multicolumn{1}{|c|}{2006} & \multicolumn{1}{|c|}{2008} \\
\hline Serra (S) & $\begin{array}{l}\text { Majorca Daily Bulletin } \\
\text { Última Hora }\end{array}$ & $\begin{array}{l}\text { Majorca Daily Bulletin } \\
\text { Última Hora }\end{array}$ & $\begin{array}{l}\text { Majorca Daily Bulletin } \\
\text { Ultima Hora }\end{array}$ \\
\hline Unidad Editorial (UNE) & $\begin{array}{l}\text { El Mundo El Día de Baleares } \\
\text { El Mundo S. Veintiuno }\end{array}$ & $\begin{array}{l}\text { El Mundo S. Veintiuno } \\
\text { Estadio Deportivo } \\
\text { Expansión } \\
\text { Marca }\end{array}$ & $\begin{array}{l}\text { El Mundo S. Veintiuno } \\
\text { Estadio Deportivo } \\
\text { Expansión } \\
\text { Vocento (V) }\end{array}$ \\
& $\begin{array}{l}\text { ABC } \\
\text { Diario Sur } \\
\text { El Comercio } \\
\text { El Correo Español } \\
\text { El Diario Montañés } \\
\text { El Diario Vasco } \\
\text { El Norte de Castilla } \\
\text { Hoy Diario de Extremadura } \\
\text { Ideal } \\
\text { La Rioja } \\
\text { La Verdad } \\
\text { La Voz de Cádiz } \\
\text { Las Provincias }\end{array}$ & $\begin{array}{l}\text { El Comercio } \\
\text { El Correo Español } \\
\text { El Diario Montañés } \\
\text { El Diario Vasco } \\
\text { El Norte de Castilla } \\
\text { Hoy Diario de Extremadura } \\
\text { Ideal } \\
\text { La Rioja } \\
\text { La Verdad } \\
\text { La Voz de Cádiz } \\
\text { Las Provincias } \\
\text { La Voz de Avilés }\end{array}$ & $\begin{array}{l}\text { ABC } \\
\text { Diario Sur } \\
\text { El Comercio } \\
\text { El Correo Español } \\
\text { El Diario Montañés } \\
\text { El Diario Vasco } \\
\text { El Norte de Castilla } \\
\text { Hoy Diario de Extremadura } \\
\text { Ideal } \\
\text { La Rioja } \\
\text { La Verdad } \\
\text { La Voz de Cádiz } \\
\text { Las Provincias } \\
\text { Qué! }\end{array}$ \\
\hline
\end{tabular}

4 El Mundo El Día de Baleares no es analizado en 2008 porque se integra en la versión nacional como una sección más y no se diferencia dentro de la Web como una cabecera específica.

5 La Voz de Avilés, alojada en la Web de El Comercio, presenta una cabecera diferenciada y se analizan sólo aquellas secciones y/o espacios que se identifica bajo este diario. Aparece entre los medios auditados por la OJD pero no ofrece datos de difusión en 2007. 


\begin{tabular}{|c|c|c|c|}
\hline Grupo & 2006 & 2007 & 2008 \\
\hline Zeta (Z) & $\begin{array}{l}\text { Córdoba } \\
\text { Diario Equipo } \\
\text { El Periódico de Aragón } \\
\text { El Periódico de Catalunya } \\
\text { El Periódico de Extremadura } \\
\text { El Periódico La Voz de Asturias } \\
\text { El Periódico Mediterráneo } \\
\text { Sport }\end{array}$ & $\begin{array}{l}\text { Córdoba } \\
\text { Diario Equipo } \\
\text { El Periódico de Aragón } \\
\text { El Periódico de Catalunya } \\
\text { El Periódico de Extremadura } \\
\text { El Periódico La Voz de Asturias } \\
\text { El Periódico Mediterráneo } \\
\text { Sport }\end{array}$ & $\begin{array}{l}\text { Córdoba } \\
\text { Diario Equipo } \\
\text { El Periódico de Aragón } \\
\text { El Periódico de Catalunya } \\
\text { El Periódico de Extremadura } \\
\text { El Periódico La Voz de Asturias } \\
\text { El Periódico Mediterráneo } \\
\text { Sport }\end{array}$ \\
\hline
\end{tabular}

Fuente: Elaboración propia

El programa de codificación empleado se basa en un estudio inédito de 2004 dirigido por la doctora Elvira García de Torres y, una vez adaptado, la ficha queda configurada por 70 ítems entre variables y subvariables. De ellos 16 corresponden a variables de la interactividad (wiki, encuestas, enlaces, blog, valoración, dato de lectura, cartas al director, comentarios, compartir contenidos del diario con otro usuario por email, contacto, foro, chats y/o encuentros digitales, móvil proactivo, archivo, añadir información -videos, fotos, audio, noticias,....- y la personalización), y 7 a plataformas de distribución (sindicación de contenidos, titulares por correo, mensaje a móvil, Wap, Pda, boletines por email, descargas en pdf). Se analiza el medio con especial atención a las noticias de portada y los contenidos de opinión (columnas, artículos,... ${ }^{6}$ que aparecen en la Home o en la sección específica para dar respuesta a alguno de los ítems examinados?

\section{Resultados: Incorporación de recursos y semejanzas internas en los grupos de comunicación españoles}

Se registra un incremento en la presencia de recursos en el conjunto de diarios analizados. Los porcentajes obtenidos en interactividad pasan de representar el $47 \%$ en 2006, al 54,2\% en 2007, hasta llegar al 62,5\% en 2008. En el caso de las plataformas de distribución los valores son inferiores: en 2006 la cifra obtenida es del 23,53\%, del 35,65\% en 2007 y del 38,29\% en 2008.

\footnotetext{
6 No se incluyen en este análisis, por ejemplo, las entradas de los blogs.

No se analizan los contenidos en pdf ni los visores que permiten acceder a la versión impresa del medio.
} 
A partir de los datos de cada grupo se establecen los grados de aprovechamiento de los recursos y se establece un nivel bajo (entre 0 y 33\%), medio (del 34\% hasta el 66\%) y alto (del 67\% al 100\%). La evolución positiva se registra al pasar, en el caso de la interactividad, la mayor parte de los grupos del nivel medio a localizarse, en 2008, el 50\% en el estadio superior. En las plataformas de distribución la evolución es evidente entre 2007 y 2008 por el descenso de grupos en el nivel bajo. No obstante, en 2008 siguen en ese estadio seis de los doce grupos analizados. Destaca que Prisa es el único que alcanza el nivel superior desde 2007 tanto en interactividad como en plataformas de distribución.

El 75\% de los grupos experimentan un incremento en el aprovechamiento de aplicaciones interactivas entre 2006 y 2007. El porcentaje es del 91,67\% en el paso de 2007 a 2008. Un grupo en cada periodo registra un descenso en los resultados: Unedisa entre 2006 y 2007 y Promecal entre 2007 y 2008. Solo Serra y La Región ofrecen los mismos porcentajes de recursos en 2006 y 2007. En el caso de las plataformas de distribución la tendencia dominante es también el crecimiento (presente en el 58,33\% de los grupos) que se mantiene invariable en el periodo estudiado. Solo entre 2007 y 2008 se observa un descenso en los datos de Prisa y Promecal. Un 41,67\% de los grupos presenta el mismo porcentaje de plataformas de distribución en 2006 y 2007; el dato es inferior en la segunda etapa (un 25\%). Los grupos de comunicación muestran algunas divergencias internas pues algunas cabeceras destacan en la incorporación de herramientas frente al resto de diarios de la compañía. Son especialmente significativos por liderar en solitario en sus respectivos grupos durante todo el periodo analizado tanto en interactividad como en plataformas El Mundo en Unidad Editorial y La Vanguardia en Godó.

Tabla 2. Oferta de los recursos en los grupos de comunicación: niveles de implementación en términos porcentuales (2006-2008)

\begin{tabular}{|c|c|c|c|c|c|c|}
\hline & \multicolumn{3}{|c|}{ Interactividad } & \multicolumn{3}{|c|}{ Plataformas de distribución } \\
\hline Niveles & 2006 & 2007 & 2008 & 2006 & 2007 & 2008 \\
\hline $\begin{array}{l}\text { Alto (de } 67 \% \text { a } \\
100 \%)\end{array}$ & & $\begin{array}{l}\mathrm{P}(75) \\
\mathrm{V}(70,5)\end{array}$ & $\begin{array}{l}\text { P }(81,3) \\
\text { V }(77,2) \\
\text { EPI }(70,3) \\
\text { GD }(68,8) \\
J(67,4) \\
Z(67,2)\end{array}$ & & $\mathrm{P}(85,7)$ & $\mathrm{P}(81)$ \\
\hline
\end{tabular}




\begin{tabular}{|c|c|c|c|c|c|c|}
\hline & \multicolumn{3}{|c|}{ Interactividad } & \multicolumn{3}{|c|}{ Plataformas de distribución } \\
\hline Niveles & 2006 & 2007 & 2008 & 2006 & 2007 & 2008 \\
\hline $\begin{array}{l}\text { Medio } \\
\text { (de } 34 \% \text { a } 66 \% \text { ) }\end{array}$ & $\begin{array}{l}\text { V }(65,9) \\
Z(53,9) \\
\text { UNE(53,1) } \\
\text { GD }(50) \\
\text { EPI }(46,9) \\
\text { P }(44,8) \\
\text { PG }(40,6) \\
\text { J }(35,9) \\
\text { LR }(34,4)\end{array}$ & $\begin{array}{l}\text { Z }(60,9) \\
\text { GD }(53,1) \\
J(52,3) \\
\text { EPI }(51,6) \\
\text { PG }(50) \\
\text { UNE (50) } \\
\text { H }(34,4) \\
\text { LR }(34,4)\end{array}$ & $\begin{array}{l}\text { PG }(64,6) \\
\text { LR }(62,5) \\
\text { H }(56,3) \\
\text { UNE }(51,6)\end{array}$ & $\begin{array}{l}\mathrm{P}(47,6) \\
\mathrm{UNE}(42,9) \\
\mathrm{V}(40,7) \\
\mathrm{GD}(35,7)\end{array}$ & $\begin{array}{l}\text { V (51) } \\
\text { GD (50) } \\
\text { UNE }(42,9)\end{array}$ & $\begin{array}{l}\mathrm{V}(61,2) \\
\mathrm{UNE}(50) \\
\mathrm{GD}(50) \\
\mathrm{Z}(44,6) \\
\mathrm{J}(42,9)\end{array}$ \\
\hline $\begin{array}{l}\text { Bajo } \\
\text { (de } 0 \text { a 33\%) }\end{array}$ & $\begin{array}{l}\text { H }(31,3) \\
\text { PR }(27,5) \\
\text { S }(21,9)\end{array}$ & $\begin{array}{l}\text { PR }(32,5) \\
S(21,9)\end{array}$ & $\begin{array}{l}\text { PR }(26,9) \\
\text { S (25) }\end{array}$ & $\begin{array}{l}\text { PG }(28,6) \\
\text { EPI(21,4) } \\
\text { H }(21,4) \\
\text { Z }(19,6) \\
\text { LR }(14,3) \\
J(0) \\
\text { PR (0) } \\
\text { S (0) }\end{array}$ & \begin{tabular}{|l} 
Z (32,1) \\
PG $(28,6)$ \\
EPI $(26,8)$ \\
H $(21,4)$ \\
J $(14,3)$ \\
LR $(14,3)$ \\
PR $(8,6)$ \\
S (0)
\end{tabular} & $\begin{array}{l}\text { EPI }(30,4) \\
\text { H }(28,6) \\
\text { PG }(28,6) \\
\text { LR }(28,6) \\
\text { PR }(4,3) \\
\text { S (0) }\end{array}$ \\
\hline
\end{tabular}

Fuente: Elaboración propia

Siguiendo el principio aplicado en el estudio realizado por García de Torres, Rodríguez Martínez y Martínez Martínez (2010), la sintonía en la oferta de recursos se plantea en términos generales tanto por la presencia como por la ausencia de herramientas en todas las cabeceras analizadas de cada grupo de comunicación. En 2006, en interactividad, destaca la correspondencia alcanzada en las cabeceras del grupo Joly (con un 93,75\%, es decir, hay coincidencia en 15 de los 16 recursos estudiados); en 2007, con el 81,25\% se encuentran Prisa, Joly, Grupo Heraldo y Serra; aunque el resultado más alto, el 100\%, lo presenta La Región en 2008. Porcentajes elevados se observan en el estudio de las plataformas de distribución donde se alcanzan valores del 100\% durante los tres años en que se practica el análisis: en 2006, en Joly, Serra y Promecal; en 2007, en Prisa, Serra y Promecal y, en 2008, en Joly, Serra, El Progreso y La Región. La mayor sintonía registrada en estos grupos se presenta por la ausencia de variables salvo en Prisa que además muestra la mayor evolución al pasar del 0\% registrado en 2006 a la correspondencia total de 2007. Solo otro grupo registra una disparidad total en la oferta y es Unidad Editorial en 2007. 
Predomina el nivel alto de sintonía (que incluye grupos de comunicación con porcentajes de entre el $67 \%$ y el 100\%) pues en los tres años, tanto en el estudio de las plataformas de distribución como en la interactividad, se registra que el 50\% o más de los grupos analizados se encuentran en este estadio. No obstante, las variaciones registradas en las cifras obtenidas por las compañías no muestran un patrón claro de evolución. En el análisis de interactividad, mientras que entre 2006 y 2007 desciende la sintonía registrada en ocho de los doce grupos analizados, en el paso de 2007 a 2008 se observa un incremento en el 50\% de los casos. En cuanto a las plataformas de distribución se obtiene una mayor estabilidad puesto que si en la primera etapa el 58,33\% presenta los mismos datos de sintonía en términos porcentuales, en la segunda esta tendencia sigue siendo mayoritaria al encontrarse en el 41,67\% de los grupos.

Tabla 3. Presencia y ausencia de las herramientas en las cabeceras del grupo. Datos del número de variables

\begin{tabular}{|c|c|c|c|c|c|c|c|}
\hline \multirow{2}{*}{ Grupo } & \multirow{2}{*}{ Tipo } & \multicolumn{2}{|c|}{2006} & \multicolumn{2}{|c|}{2007} & \multicolumn{2}{|c|}{2008} \\
\hline & & Todos & Ninguno & Todos & Ninguno & Todos & Ninguno \\
\hline \multirow{2}{*}{ V } & Interactividad & 9 & 3 & 9 & 2 & 8 & 1 \\
\hline & Plataformas de distribución & 0 & 1 & 1 & 0 & 1 & 0 \\
\hline \multirow{2}{*}{$\mathrm{P}$} & Interactividad & 0 & 3 & 11 & 2 & 12 & 2 \\
\hline & Plataformas de distribución & 0 & 0 & 6 & 1 & 5 & 1 \\
\hline \multirow{2}{*}{ UNE } & Interactividad & 5 & 4 & 2 & 3 & 2 & 3 \\
\hline & Plataformas de distribución & 0 & 1 & 0 & 0 & 0 & 1 \\
\hline \multirow{2}{*}{ GD } & Interactividad & 7 & 7 & 6 & 5 & 9 & 3 \\
\hline & Plataformas de distribución & 1 & 3 & 2 & 2 & 2 & 2 \\
\hline \multirow{2}{*}{ EPI } & Interactividad & 4 & 5 & 3 & 4 & 8 & 4 \\
\hline & Plataformas de distribución & 0 & 4 & 1 & 4 & 2 & 4 \\
\hline \multirow{2}{*}{$\mathrm{Z}$} & Interactividad & 6 & 3 & 7 & 3 & 8 & 4 \\
\hline & Plataformas de distribución & 0 & 3 & 1 & 3 & 1 & 1 \\
\hline \multirow{2}{*}{$\mathrm{J}$} & Interactividad & 5 & 10 & 8 & 5 & 9 & 4 \\
\hline & Plataformas de distribución & 0 & 7 & 1 & 6 & 3 & 4 \\
\hline \multirow{2}{*}{$\mathrm{H}$} & Interactividad & 4 & 10 & 4 & 9 & 6 & 4 \\
\hline & Plataformas de distribución & 1 & 5 & 1 & 5 & 1 & 4 \\
\hline
\end{tabular}




\begin{tabular}{|l|l|c|c|c|c|c|c|}
\hline \multirow{2}{*}{ Grupo } & \multirow{2}{*}{ Tipo } & \multicolumn{2}{|c|}{2006} & \multicolumn{2}{|c|}{2007} & \multicolumn{2}{c|}{2008} \\
\cline { 3 - 8 } & & Todos & Ninguno & Todos & Ninguno & Todos & Ninguno \\
\hline \multirow{2}{*}{ S } & Interactividad & 2 & 11 & 2 & 11 & 2 & 10 \\
\cline { 2 - 9 } & Plataformas de distribución & 0 & 7 & 0 & 7 & 0 & 7 \\
\hline \multirow{2}{*}{ PR } & Interactividad & 3 & 9 & 2 & 7 & 2 & 6 \\
\cline { 2 - 9 } & Plataformas de distribución & 0 & 7 & 0 & 4 & 0 & 4 \\
\hline \multirow{2}{*}{ PG } & Interactividad & 4 & 7 & 4 & 4 & 9 & 4 \\
\hline \multirow{2}{*}{ LR } & Plataformas de distribución & 1 & 4 & 1 & 4 & 2 & 5 \\
\cline { 2 - 9 } & Interactividad & 3 & 8 & 3 & 8 & 10 & 6 \\
\hline
\end{tabular}

Fuente: Elaboración propia

En cuanto a la homogeneidad en la oferta, es decir, la correspondencia exclusivamente en aquellas variables que sí están presentes en todos los medios de un grupo de comunicación, en 2006 registraba en el caso de la interactividad los valores máximos Vocento puesto que el 56,25\% de las 16 variables estudiadas se ofertaban en todas sus cabeceras. En 2007 y 2008 destacaba la presencia de recursos coincidentes en Prisa (con un 68,75\% y un 75\% respectivamente).

Atendiendo a los resultados de cada compañía, frente a la estabilidad observada entre 2006 y 2007 (dado que el 41,67\% de los grupos mantenía los mismos porcentajes de homogeneidad en la presencia de aplicaciones interactivas), entre 2007 y 2008 la mayoría (66,67\%) presenta un incremento. En el conjunto de grupos la evolución es positiva (véase el gráfico 2): si en 2006 y 2007 el mayor porcentaje se situaba en niveles bajos de homogeneidad (con 9 y 7 grupos respectivamente que presentaban entre $0 \%$ o el $33 \%$ de las variables analizadas en todos sus periódicos), en 2008 la mayoría (ocho de los doce grupos estudiados) se encuentra en el nivel medio. El nivel superior resulta un estadio excepcional pues solo Prisa ${ }^{8}$ presenta este nivel de uniformidad.

8 Destaca el hecho de que Prisa parte de niveles de homogeneidad del 0\% en 2006. Aunque en ese año uno de los diarios, Odiel Información, se encontraba en construcción y es significativo de los distintos niveles de desarrollo de las cabeceras del grupo, tampoco altera significativamente los resultados obtenidos en ese periodo pues en el resto de cabeceras solo se registra coincidencia en 2 de las 16 variables por lo que seguiría en niveles inferiores. 
Figural. Grupos de comunicación según el nivel de homogeneidad en la presencia de variables interactivas (\%)

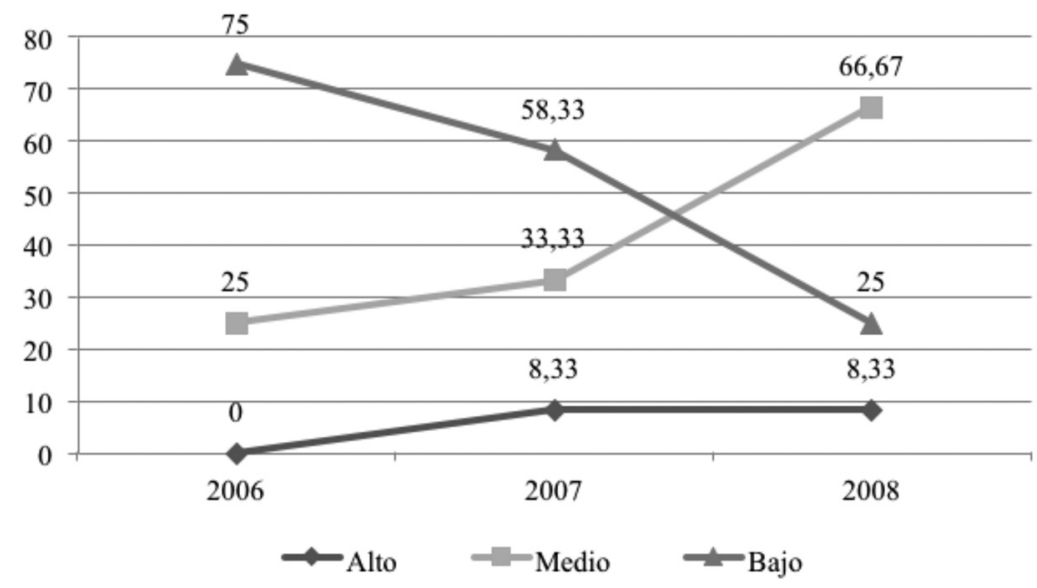

Fuente: Elaboración propia

Son tres los grupos que presentan homogeneidad en la presencia de las plataformas de distribución estudiadas en 2006. Se trata de Godó, Grupo Heraldo y El Progreso que tienen un 14,29\% de correspondencia sobre las 7 herramientas analizadas. En 2007 y 2008 Prisa vuelve a destacar obteniendo valores del 85,71\% y 71, 43\% respectivamente.

Según los datos de homogeneidad obtenidos de cada grupo, se registra un estancamiento de las cifras entre 2006 y 2007 en el 50\% de los grupos y entre 2007 y 2008 en el 58,33\%. Esta tendencia contrasta con los elevados datos de crecimiento que también se observan en el periodo: un 50\% y un 33,33\% respectivamente. Predomina una baja homogeneidad en la presencia de plataformas de distribución aunque porcentualmente se registra un ligero descenso en este nivel: mientras que en 2006 la totalidad de los grupos analizados se encontraba en esa situación, en 2008 serán diez. Solo Prisa alcanza el nivel superior en 2007 y 2008 y Joly el intermedio al final del periodo. 
Figura 2. Grupos de comunicación según el nivel de homogeneidad en la presencia de plataformas de distribución (\%)

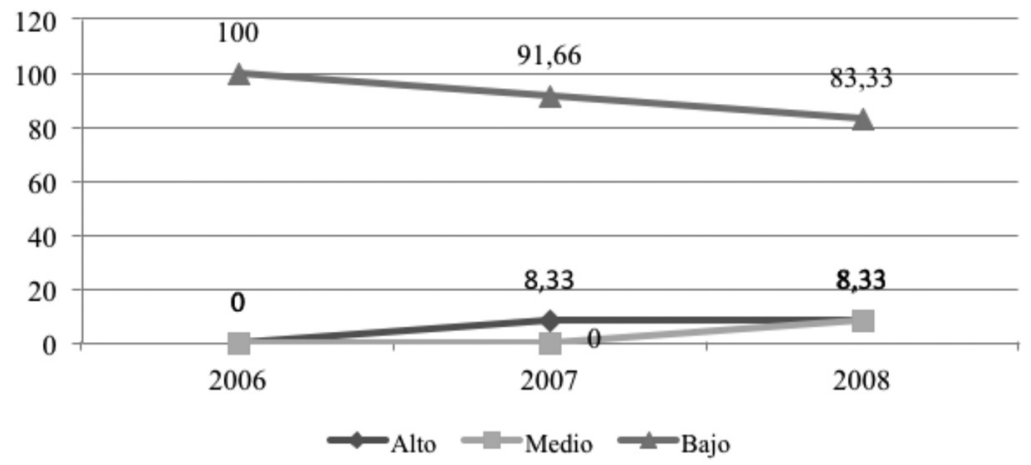

Fuente: Elaboración propia

Serra es el grupo que mantiene los mismos resultados en toda la etapa tanto en interactividad como en plataformas. Mayor estabilidad se registra precisamente en el análisis de estas últimas herramientas pues también el Grupo Heraldo y El Progreso presentan los mismos datos durante los tres años.

\subsection{Estudio de la popularidad de las herramientas entre los principales grupos de comunicación}

El análisis de las similitudes permite establecer tendencias en la popularidad de las herramientas que son incluidas en todos los diarios de la mayoría de los grupos. Así, el contacto, el archivo y los enlaces en los contenidos son los recursos más destacados. El primero llega incluso en 2007 a registrarse en todos los diarios de los doce grupos analizados. En el lado opuesto (es decir, ningún grupo apostaba por ofrecer la herramienta en alguna de las cabeceras analizadas) figura la posibilidad de comentar los contenidos en 2006, los wikis en 2007, o 'el móvil proactivo' con publicación de mensajes en portada en 2008. En las plataformas los resultados más elevados de correspondencia se registran en la ausencia de las herramientas destacando en 2007 el envío de textos al usuario por correo.

Tabla 4. Herramientas que presentan todas o ninguna de las cabeceras de los principales grupos de comunicación (2006-2008): datos del número de compañías

\begin{tabular}{|l|c|c|c|c|c|c|}
\hline & \multicolumn{2}{|c|}{2006} & \multicolumn{2}{c|}{2007} & \multicolumn{3}{c|}{2008} \\
\hline Herramientas Interactividad & Todos & Ninguno & Todos & Ninguno & Todos & Ninguno \\
\hline Contacto & 11 & 0 & 12 & 0 & 11 & 0 \\
\hline Archivo & 10 & 0 & 11 & 0 & 11 & 0 \\
\hline
\end{tabular}




\begin{tabular}{|c|c|c|c|c|c|c|}
\hline \multirow[b]{2}{*}{ Herramientas Interactividad } & \multicolumn{2}{|c|}{2006} & \multicolumn{2}{|c|}{2007} & \multicolumn{2}{|c|}{2008} \\
\hline & Todos & Ninguno & Todos & Ninguno & Todos & Ninguno \\
\hline Enlaces en los contenidos & 9 & 0 & 9 & 0 & 11 & 0 \\
\hline Encuestas & 8 & 1 & 8 & 1 & 9 & 0 \\
\hline Envío de contenidos a un amigo por email & 3 & 1 & 5 & 1 & 9 & 1 \\
\hline Weblogs & 1 & 4 & 2 & 3 & 7 & 2 \\
\hline Valorar los contenidos & 0 & 9 & 3 & 5 & 5 & 3 \\
\hline Comentar los contenidos & 0 & 12 & 1 & 4 & 5 & 1 \\
\hline Añadir información & 2 & 5 & 2 & 2 & 5 & 1 \\
\hline Chats y encuentros digitales & 2 & 3 & 3 & 5 & 5 & 0 \\
\hline Dato de lectura en los contenidos & 0 & 10 & 1 & 9 & 2 & 9 \\
\hline Publicación de cartas al director & 1 & 3 & 2 & 1 & 2 & 2 \\
\hline Foros & 4 & 3 & 2 & 2 & 2 & 3 \\
\hline Personalización & 1 & 7 & 0 & 7 & 1 & 6 \\
\hline Wikis & 0 & 11 & 0 & 12 & 0 & 11 \\
\hline Móvil proactivo & 0 & 11 & 0 & 11 & 0 & 12 \\
\hline Sindicación de contenidos & 0 & 4 & 4 & 3 & 8 & 1 \\
\hline Contenidos del medio impreso en PDF & 2 & 3 & 5 & 1 & 5 & 2 \\
\hline Textos por correo & 0 & 8 & 0 & 10 & 2 & 7 \\
\hline Envío de contenidos al móvil & 0 & 9 & 2 & 8 & 2 & 7 \\
\hline Titulares por correo & 0 & 5 & 1 & 6 & 1 & 6 \\
\hline Móvil. Navegación WAP & 0 & 8 & 1 & 6 & 1 & 7 \\
\hline PDA & 0 & 7 & 1 & 7 & 0 & 8 \\
\hline
\end{tabular}

Fuente: Elaboración propia

Analizando la unanimidad en la presencia de recursos interactivos entre las cabeceras de cada compañía, la mayoría de las herramientas solo alcanzan un bajo nivel de implementación (entre 0 y 4 grupos). En ese estadio figuran el 75\% de las herramientas en 2006, el 68,75\% en 2007 y el 37,5\% en 2008. Por el contrario, solo tres herramientas en 2006 y 2007 y cinco 
en 2008 se encontraban en niveles superiores (entre 9 y 12 grupos). La evolución es positiva pues, entre 2006 y 2007, 9 de las aplicaciones pasan a estar presentes de forma unánime en un mayor número de grupos. Ese incremento se observa en el 62,5\% de las herramientas entre 2007 y 2008. Destaca, a lo largo del periodo, el incremento observado en el número de grupos de comunicación que apuestan por ofrecer recursos tales como el weblog, la posibilidad de que un usuario comparta con un amigo los contenidos por email, la opción de comentar y de valorar. Solo se registra un descenso en 2 recursos en la primera etapa (el foro y la personalización) y en uno en la segunda (el contacto).

En las plataformas también se observa un predominio de la baja implementación en términos de unanimidad en las cabeceras de cada grupo. En 2007 una de las aplicaciones alcanza un nivel medio, el pdf, y en 2008 ocupa esta posición junto a la sindicación de contenidos. Si la tendencia principal entre 2006 y 2007 es el crecimiento (en el 85,71\% de las herramientas se registra un aumento en el número de grupos que las ofertan en todos los diarios analizados), entre 2007 y 2008 será el estancamiento $(57,14 \%)$. Sólo una aplicación desciende en este último periodo (la Pda) al pasar de figurar en todos los diarios de uno de los grupos a que no se de esta correspondencia en ninguno.

\subsection{Análisis de las semejanzas en los recursos de interactividad selectiva y comunicativa, el UGC y la Web 2.0}

Dada la diversidad de recursos analizados en el programa de codificación, se pueden establecer patrones en la incorporación de la interactividad selectiva y comunicativa . En ambos casos los niveles de aprovechamiento se sitúan en niveles intermedios, con datos ligeramente superiores en la interactividad selectiva donde se alcanza en 2008 el 66,67\% de implementación frente al 59,26\% en la interactividad comunicativa ${ }^{9}$. La tendencia creciente se registra en todo el periodo pues la mayoría de los grupos aumentan las cifras de implementación en ambas categorías. No obstante, mientras que en la comunicativa solo dos grupos llegan a niveles altos en la oferta de aplicaciones en 2008 (Prisa con el 74,07\% y EPI con el 69,44\%), en la interactividad selectiva, en ese mismo año, la mayoría de grupos figuran en esta categoría: Prisa, Godó, EPI, Zeta, Joly, Grupo Heraldo, El Progreso y La Región. Solo Serra y Promecal muestran valores bajos.

En cuanto a la sintonía por la presencia y/o ausencia de recursos, se observa un predominio del nivel alto en las herramientas de interactividad selectiva, aunque con ligeras oscilaciones: en 2006 en este nivel se contabilizaban el 83,33\% de

\footnotetext{
9 Se emplea la terminología aplicada por Alejandro Rost (2006). En el presente estudio el elemento diferencial que justifica la clasificación reside en el sujeto con el que la aplicación facilita que se mantenga la relación. Así, la interactividad comunicativa permite una comunicación creativa con las personas por lo que engloba las siguientes herramientas que se analizan en este estudio: comentar los contenidos, las opciones de contacto, cartas al director, foros, chats, blogs, wiki, añadir contenidos, el móvil proactivo. La interactividad selectiva hace posible la relación con la prestación y/o con el contenido y de las aplicaciones analizadas en este estudio incluiría: las encuestas, el envío de contenidos a un amigo, los datos de lectura, la valoración de los contenidos, los enlaces, la personalización, y el archivo o bases de datos (Martínez Martínez, 2010).
} 
los grupos, en 2007 el 58,33\% y en 2008 el 75\%. Destaca que Zeta y Joly alcanzan valores de similitud del 100\% en 2007 y en 2008, junto a estos dos grupos, obtienen ese mismo resultado EPI, El Progreso y La Región. En la interactividad comunicativa, si en 2006 el 50\% de los grupos registraban una sintonía alta, en los años siguientes el porcentaje desciende y cobra importancia niveles medios de correspondencia (donde se encuentran en 2007 el 50\% de los grupos y en 2008 el 58,33\%). Joly y La Región presentan aquí también resultados del 100\%, en 2006 y 2008 respectivamente. En 2007, cuatro son los grupos que comparten resultados máximos (el 77,78\%): Vocento, Prisa, Grupo Heraldo y Serra.

Atendiendo de forma exclusiva a la homogeneidad en la presencia de variables, destaca el incremento del nivel alto de correspondencia en la interactividad selectiva (es decir, grupos que presentan en todos sus diarios cinco o más de las siete variables analizadas) pues pasa de no tener representación en 2006 a alcanzar ese nivel la mitad de los grupos. Los datos más elevados los ofrecen en 2006 Vocento, Unedisa y Zeta (con la presencia de cuatro de las herramientas en todas sus cabeceras); en 2007, Prisa (con seis de los recursos); y, en 2008, junto a Prisa figura El Progreso también con seis de las variables analizadas. Algunos de estos grupos presentan en otros periodos las cifras más bajas: en 2006 en Prisa no se registra la presencia de ninguna de las aplicaciones en todos sus diarios; en 2007, junto a Serra y Promecal -que repetirán posiciones en 2008-, Unidad Editorial registra niveles de homogeneidad del 14,28\%. Las tendencias mayoritarias son la estabilidad y el incremento de correspondencia. Entre 2007 y 2008 ningún grupo presenta un descenso los resultados de homogeneidad registrados en esta tipología. En el caso de la interactividad comunicativa, ningún grupo alcanza una alta homogeneidad. Los bajos niveles son la tónica dominante en el periodo. No se encuentra correspondencia en la presencia de recursos de interactividad selectiva ni en Prisa en 2006 ni en Unidad Editorial en 2008. Los valores máximos, los presenta Godó en 2006 (44,44\%); en 2007, Vocento (55.56\%) y Prisa (55,56\%) que repetirá posición en 2008 aunque con resultados más altos (66,67\%). Además de este, otros siete grupos presentan resultados porcentuales superiores en el último año estudiado respecto a los mostrados el curso anterior. Si en la primera etapa la tendencia es el estancamiento pues el $66,67 \%$ de los grupos registran el mismo número de variables presentes en todas sus cabeceras en 2006 y 2007, en la segunda ocho de los doce grupos muestra un crecimiento en los resultados. 
Figura 3. Grupos de comunicación según el nivel de homogeneidad en la presencia de variables de interactividad selectiva (\%)

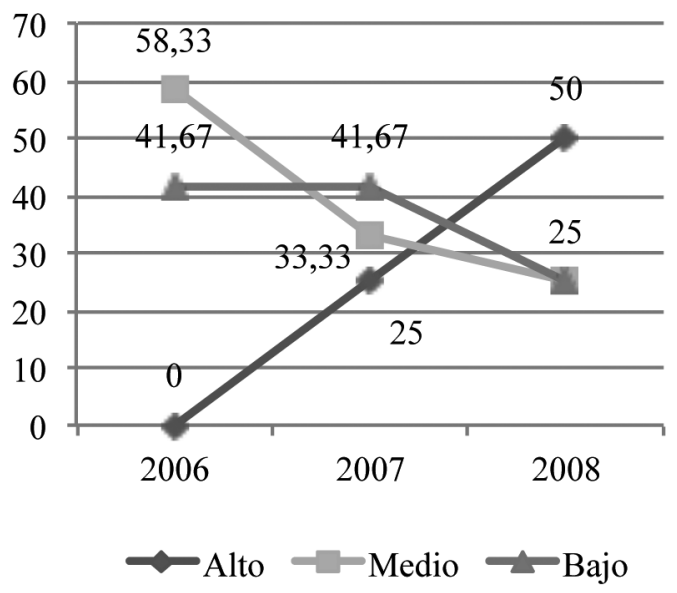

Fuente: Elaboración propia
Figura 4. Grupos de comunicación según el nivel de homogeneidad en la presencia de variables de interactividad comunicativa (\%)

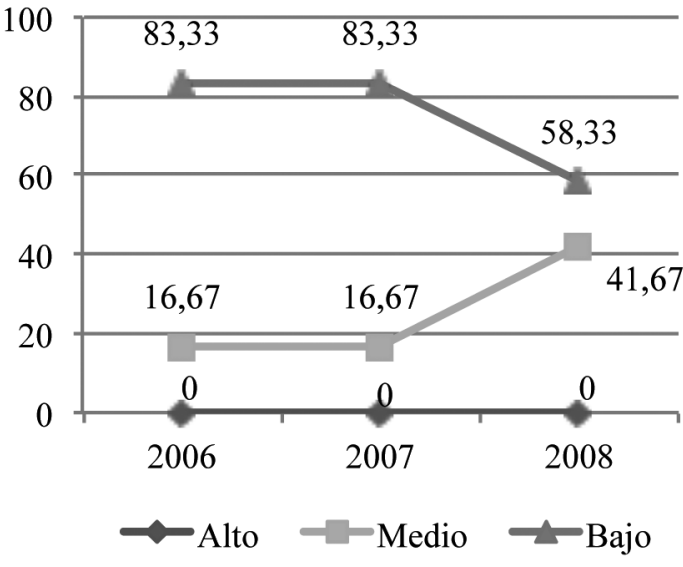

Fuente: Elaboración propia

De un subgrupo creado a partir de las 16 variables de interactividad se puede comprobar la evolución en la incorporación de aplicaciones del UGC. Se incluyen los siguientes recursos: encuestas, valoración de los contenidos, datos de lectura, comentar contenidos, cartas al director, foros, chats, blogs, wikis, publicar contenidos (fotos, vídeos, frases, eventos,...) y los mensajes en portada enviados por medio del móvil. La evolución marcada es positiva: si en 2007 el $75 \%$ presentan mayores datos de implementación que en el curso anterior, en 2008 esta cifra se ve incrementada dado que once de los doce analizados aumentan sus resultados. Promecal es el único grupo que presenta un descenso en el último año y Serra muestra los porcentajes más bajos de aprovechamiento del UGC. En el lado opuesto, Vocento en 2006 y Prisa en 2007 y 2008 tienen los resultados más elevados y son los únicos que alcanzan un nivel alto de oferta de UGC hacia el final de la etapa.

Los mayores porcentajes de sintonía en UGC se obtienen por la similitud en la ausencia de variables. En 2006, en Godó, Joly y Grupo Heraldo la correspondencia alcanza aproximadamente al 91\% de las aplicaciones; en 2007, con el 81,82\%, destaca el Grupo Heraldo, Serra y Prisa; y, en 2008, con el 100\% se encuentra La Región. Solo Prisa, alcanza estas posiciones por el mayor protagonismo de las herramientas que ofrece en todos sus diarios (siete de las once analizadas) frente a la ausencia de solo dos de las aplicaciones en todos sus periódicos. Salvo en 2007, donde predomina el nivel medio de sintonía (en 58,33\% de los grupos), el resto del periodo registra mayor presencia del nivel alto (50\%) lo que supone la coincidencia en ocho o más de las herramientas. 
Como muestra el gráfico 6, sólo una compañía consigue situarse en el nivel alto de homogeneidad en la presencia de recursos de UGC. Este hito lo logra Prisa en 2008 pues ocho de las once herramientas analizadas están en los tres diarios integrados en su estructura estudiados ese periodo. El nivel bajo es el dominante salvo en el último año donde es superado por el porcentaje de grupos que presentan un nivel medio. En todo el periodo analizado existen grupos en los no se contabilizan herramientas con presencia en todos sus periódicos. Es el caso en 2006 de Prisa y Serra; en 2007, de Unidad Editorial, EPI, Serra y Promecal; y, en 2008, de Serra, Unidad Editorial y Promecal.

Figura 5. Grupos de comunicación según el nivel de homogeneidad en la presencia de variables de UGC (\%)

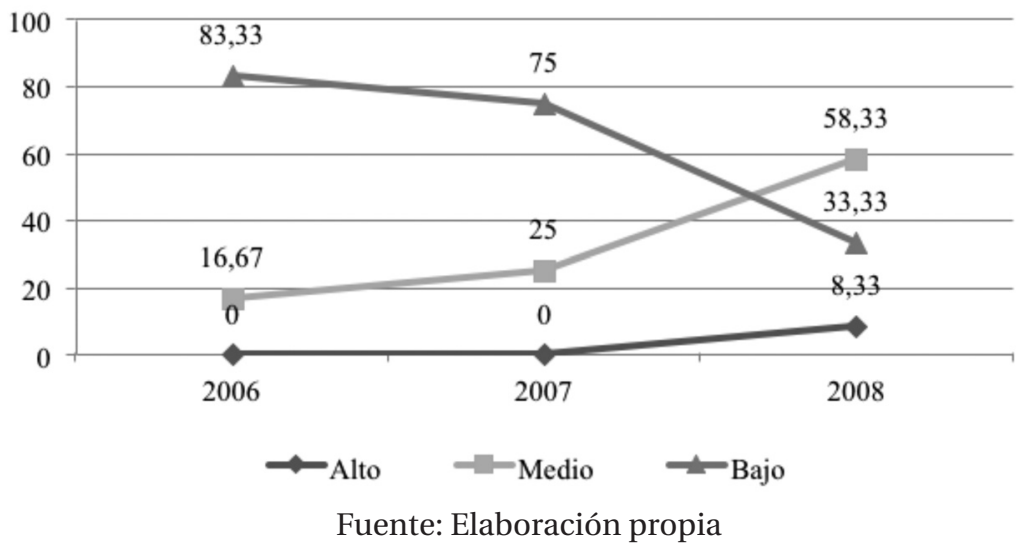

El análisis combinado de variables y subvariables tanto de la interactividad como de las plataformas de distribución permite establecer los patrones desarrollados en el caso de la Web 2.0. Partiendo de estudios previos (The Binvings Group, 2007; García de Torres et al., 2009) se establece una primera selección de recursos que se completa con la integración de otros recursos que se analizan en esta investigación y que son característicos de la Web 2.0. Son trece los ítems estudiados en este caso: enviar el contenido por email, valorar, comentar, configuración de un espacio personal, foro, chat, blog, wiki, publicar mensajes y/o contenidos, acceso a través de navegación wap, consulta en PDA, RSS, podcast. Los resultados muestran una tendencia creciente en los datos de implementación de estos recursos 2.0 pues mientras en el primer periodo nueve grupos presentan un incremento de datos, en el segundo son todos menos el grupo Serra que tiene el mismo porcentaje de aprovechamiento a lo largo de todo el periodo (3,85\%). Mientras que en 2006 y 2007 el predominio es el nivel bajo de penetración de aplicaciones (58,33\%), en 2008 se observa que en el medio (entre 5 y 8 de las herramientas) se sitúan ocho de los doce estudiados. Solo Vocento en 2008 y Prisa en 2007 y 2008 alcanzan el nivel superior de implementación.

La sintonía alta en aplicaciones 2.0 domina todo el periodo analizado y muestra una tendencia creciente al pasar de registrarse en el 50\% de los grupos en 2006 a 66,67\% en 2008. Los grupos más destacadas en sintonía son en 2006 Joly con el 
100\% de los ítems, Serra en 2007 con el 92,3\% y La Región en 2008 también con una sintonía total en herramientas 2.0. Esta correspondencia se debe principalmente a la ausencia de los recursos en todas las cabeceras de las compañías.

Como muestra el gráfico 7, ningún grupo alcanza niveles altos de homogeneidad en la presencia de estas aplicaciones. El predominio del nivel bajo es característico de 2006 y 2007 donde además se contabiliza un elevado número de compañías donde no se ofrece ni una sola de las herramientas 2.0 analizadas en todos los diarios. Es el caso de Prisa, Unidad Editorial, Grupo Heraldo, Serra, Promecal, El Progreso y La Región en 2006 y EPI, Grupo Heraldo, El Progreso, La Región, Serra, Promecal y Unidad Editorial en 2007. Estos tres últimos mantendrán los mismos resultados en 2008. Es significativo en la evolución del sector que la mayor homogeneidad se alcanza con cinco recursos en 2006, como es el caso de Vocento, y en 2007 y 2008, con ocho en Prisa. A pesar de los datos tan bajos los movimientos registrados muestran un crecimiento de la homogeneidad en el 33,33\% de los grupos en el primer periodo y del 58,33\% en el segundo.

Figura 6. Grupos de comunicación según el nivel de homogeneidad en la presencia de variables de web 2.0 (\%)

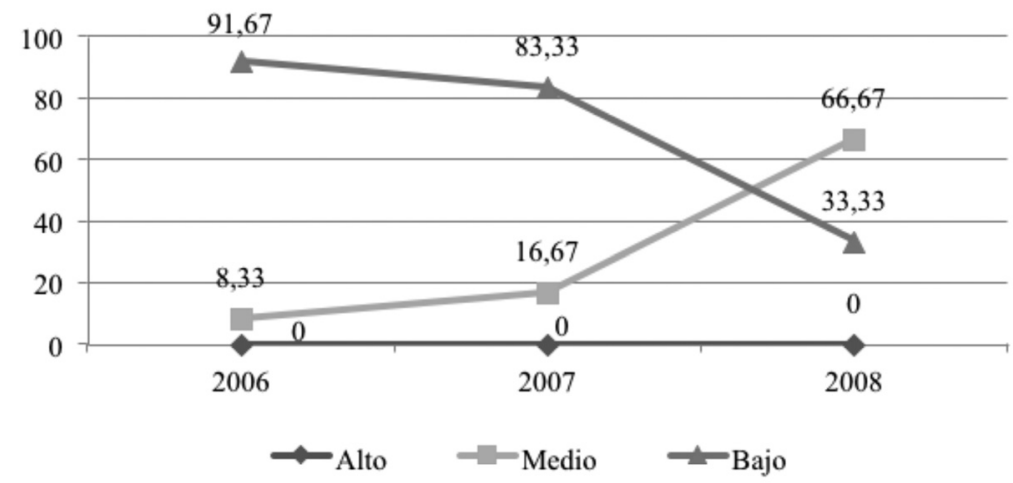

Fuente: Elaboración propia

De las tres subvariables analizadas, la configuración de un espacio personal y el podcast registran una sintonía elevada especialmente por su ausencia. Ningún grupo presenta homogeneidad en la presencia de estas aplicaciones en todos sus diarios. El RSS en 2006 tampoco se observaba en todos los diarios de una misma compañía, no obstante, conforme avanza el periodo, la implementación de este recurso se registra de forma unánime en los diarios de ocho de los grupos analizados.

\subsection{La interactividad en los contenidos: semejanzas en la oferta de herramientas en las noticias y en la opinión}

Las subvariables observadas al analizar recursos de interactividad en los contenidos permiten distinguir la presencia de ocho de las herramientas en las noticias de portada y en los textos de opinión de la Home o de la sección específica. Aun- 
que la implementación es superior en el caso de los contenidos informativos, los porcentajes de aprovechamiento alcanzados no superan el 50\%. Si bien se registra crecimiento en ambos casos, en términos porcentuales se acrecientan las diferencias entre la interactividad en las noticias y en la opinión. Sólo Prisa alcanza en 2008 un nivel alto de implementación en el caso de los recursos que acompañan a las informaciones.

La sintonía alta predomina en la interactividad en la opinión aunque también adquiere una posición destacada en el caso de la información donde solo en 2008 hay un mayor número de grupos en el nivel medio (el 50\%) frente al porcentaje alcanzado en el superior (41,67\%). En esta última tipología Joly en 2006 y La Región en 2008 obtienen una sintonía absoluta en sus cabeceras (con el 100\% de las herramientas); en el caso de la opinión estos resultados máximos los alcanzarán Godó en 2006 y Joly en 2008.

Una situación inversa se registra al observar la homogeneidad pues el periodo analizado se caracteriza por los bajos niveles obtenidos y que en 2008 en la interactividad en las noticias logra superar el grueso de grupos situados en el nivel medio. Ninguna compañía presenta un nivel alto de correspondencia en ninguna de las dos categorías analizadas ${ }^{10}$.

Figura 7. Grupos de comunicación según el nivel de homogeneidad en la presencia de variables de interactividad en las noticias (\%)

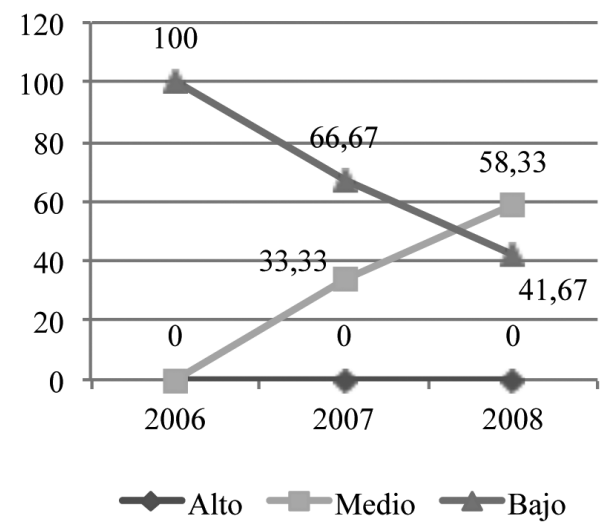

Fuente: Elaboración propia
Figura 8. Grupos de comunicación según el nivel de homogeneidad en la presencia de variables de interactividad en la opinión (\%)

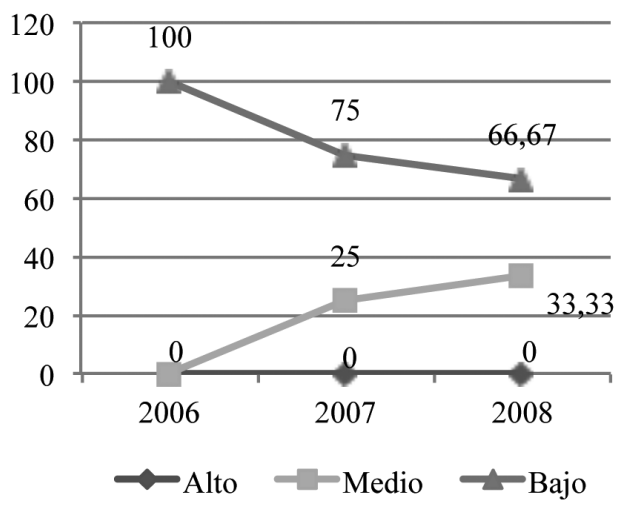

Fuente: Elaboración propia

10 Durante la etapa analizada, en el caso de las noticias, un 4,41\% de los medios estudiados en 2006 y un 2,86\% de los diarios en 2007 no ofrecían este tipo de contenidos. En total 3 de los grupos, es decir, un 25\% de las compañías analizadas, poseen algún diario que no presenta contenidos informativos en portada en alguno de los años investigados. En cuanto a la opinión, el porcentaje de medios sin ofertarlos es más elevado y pasa a ser del 8,82\% en 2006, el 11,43\% en 2007 y el 9,33\% en 2008. También es superior el número de grupos que se sitúa en el $58,33 \%$ para el periodo estudiado. 
Por herramientas, solo los enlaces internos en la noticia alcanzan índices altos de popularidad en tanto que se registran en todos los diarios de 9 de los grupos en 2006 y 2007 y en 10 en 2008. En el caso de la opinión, también esta herramienta es la más popular aunque el número de compañías en las que se registra unanimidad en la oferta se cifra en 7. Si en 2006, en ambas tipologías, 5 de las 8 aplicaciones presentaban niveles altos de sintonía en términos de ausencia pues no se ofertaban en ninguna de las cabeceras de nueve o más grupos. En el caso de la información, se mantienen en el mismo estadio en 2007 la encuesta, la valoración y, como también lo hará en 2008, el correo del autor. Entre los recursos analizados en los textos de opinión, tres de las herramientas siguen en ese nivel en los dos últimos años estudiados: la encuesta, el dato de lectura y los enlaces externos.

\section{Conclusiones}

Los resultados del estudio confirman, en términos generales, las hipótesis planteadas al inicio de la investigación. La tendencia registrada entre 2006 y 2008 es el incremento de la oferta de recursos de interactividad y plataformas de distribución en los diarios de los principales grupos de comunicación españoles. A pesar de esta evolución, solo en el caso de la interactividad y en el subgrupo de herramientas analizadas para el estudio de la interactividad selectiva, hacia al final del periodo, se observa un predominio del nivel de implementación alto (con porcentajes del $67 \%$ o más elevados) al encontrarse en este estadio la mayor parte de las compañías. Entre los recursos de UGC, Web 2.0 e interactividad comunicativa destaca el avance hacia valores medios en 2008. Por el contrario, la mayor parte de los grupos sigue registrando niveles bajos de penetración tanto en el análisis de las plataformas de distribución como de las herramientas de interactividad en las noticias y la opinión. No obstante, como ya se observara en investigaciones previas (García de Torres et al, 2004b), se comprueba la presencia de distintos ritmos en la asimilación de las aplicaciones en función del grupo e incluso la existencia de movimientos decrecientes o de estancamiento en la incorporación de variables.

El estudio de las herramientas demuestra una tendencia constante en la popularidad alcanzada por las opciones de contacto, el archivo y el hipertexto, específicamente por la oferta de enlaces internos en contenidos informativos. Destaca también la apuesta que realizan algunos grupos por incorporar en el conjunto de los diarios analizados opciones como el weblog, compartir los contenidos con otro usuario por email, el comentario y la valoración. Entre las plataformas es significativo que, conforme avanza el periodo, solo el uso del pdf y, hacia el final, la sindicación de contenidos (concretamente por los índices alcanzados en 2008 al ser ofertado en todas las cabeceras del 66,67\% de los grupos analizados) se sitúan en estadios intermedios.

Se puede hablar de una sintonía alta tanto en interactividad como en plataformas de distribución en los diarios de los principales grupos de comunicación españoles, coincidiendo con los resultados obtenidos en el análisis desarrollado por García de Torres, Rodríguez Martínez y Martínez Martínez (2010). En el caso de la interactividad comunicativa, la interac- 
tividad en la información y de los recursos de UGC se observan oscilaciones a lo largo del periodo entre los niveles superiores e intermedios. Al registrar la homogeneidad en la presencia de herramientas que ofrecen los grupos en todas sus cabeceras la tendencia en la mayoría de los grupos es mantenerse en niveles bajos en el caso de las plataformas, la interactividad comunicativa y la interactividad en la opinión. Aunque en la interactividad, la 2.0, el UGC y la interactividad en las noticias se avanza hacia niveles medios en 2008, la interactividad selectiva es la única en ese año que se presenta en la mayoría de grupos en el estadio alto.

Por grupos, aunque otras compañías lideran puntualmente algunas de las tipologías estudiadas, Joly en 2006, Prisa y Serra en 2007 y La Región en 2008 destacan por presentar los resultados de sintonía más elevados en casi todas las categorías analizadas. En el caso de la homogeneidad en la presencia de recursos, Vocento en 2006 y Prisa en 2007 y 2008 son las compañías que sobresalen por registrar los resultados más altos en la mayoría de las tipologías. La comparativa entre los resultados de implementación y los de homogeneidad permite hablar de un incremento en el desarrollo de políticas conjuntas (donde las diferencias entre ambos datos no son significativas, es decir, no superan los 10 puntos porcentuales) en el caso de las plataformas de distribución mientras que en la interactividad se registra un descenso de modo que en 2008 sólo El Progreso, La Región y Prisa cumplen con este requisito si bien con un apuesta media/alta de recursos. A pesar de las correspondencias planteadas se observa una mayor presencia de herramientas en algunos diarios en las compañías analizadas por lo que encabezan la oferta de recursos dentro del grupo.

\section{Referencias bibliográficas}

Albornoz, L. A. (2007): Periodismo digital. Los grandes diarios en la red. Buenos Aires: Ediciones La Crujía.

Almiron, N. (2009): "Grupos privados propietarios de medios de comunicación en España: principales datos estructurales y financieros”, Comunicación y Sociedad, v. 22, nºl, pp. 243-263. Disponible en: http://www.unav.es/fcom/ comunicacionysociedad/es/articulo.php?art_id=36 [Consultado el: 18 de julio de 2011]

Becerra, M.; Mastrini, G. (2006): “Marco Teórico y Metodológico”, en Becerra, M.; Mastrini, G. (Dir.): Periodistas y Magnates. Estructura y concentración de las Industrias culturales en América Latina. Buenos Aires: Prometeo, pp.37-75.

Boix Palop, A.; López García, G. (2006): “Medios de comunicación de masas y comunicación digital”, en Boix Palop, A.; López García, G. (eds.): La autoría en la era digital: Industria cultural y medios de comunicación. Valencia: Tirant lo Blanch, pp. 167-200.

Cea Esteruelas, M. N. (2009): "El área de negocio digital en los grupos de comunicación: El caso de Prisa”, Actas del I Congreso Internacional Latina de comunicación social. Tenerife: Universidad de La Laguna. Disponible en: http://www.revistalatinacs.org/09/Sociedad/actas/62nereida.pdf. Consultado el: 25 de julio de 2011. 
Díaz Noci, J. (2010): “Medios de comunicación en Internet: algunas tendencias”, El Profesional de la Información, v.19, n.6, noviembre-diciembre 2010, pp. 561-567.

Domingo, D. et al. (2007): "Four Dimensions of Journalistic Convergence: A preliminary approach to current media trends at Spain", 8th International Symposium of Online Journalism, Austin, 30-31 de marzo de 2007. Disponible en http://online.journalism.utexas.edu/2007/papers/Domingo.pdf. Consultado el: 25 de julio de 2011.

Domingo, D. et al. (2008): "Participatory journalism practices in the media and beyond : an international comparative study of initiatives in online newspapers", Journalism practice, 2 (3), pp. 326-342.

García de Torres, E.; Rodríguez Martínez, J. y Martínez Martínez, S. (2010). "Cabeceras en sintonía. Estudio de las herramientas 2.0 en los grupos de prensa españoles”, en Said Hung, E. (Ed.): TIC, comunicación y periodismo digital. Tomo II. Escenarios de enseñanza y ciberperiodismo. Barranquillla: Ediciones Uninorte, pp. 225-246.

García de Torres, E. et al. (2009). "UGC Status and Levels of Control in Argentine, Colombian, Mexican, Peruvian, Portuguese, Spanish, US and Venezuelan Online Newspapers", 10th International Symposium on Online Journalism, Austin, 17-18 de abril de 2009. Disponible en: http://online.journalism.utexas.edu/2009/papers/Garciaetal09.pdf. [Consultado el: 20 de junio de 2010].

García de Torres, E., et al. (2008): “Las herramientas 2.0 en los diarios españoles 2006-2008”, Prisma.com, 6. Disponible en: http://prisma.cetac.up.pt/193_Las_Herramientas_2.0_en_los_diarios_espanoles_2006_2008_tendencias_Elvira_Torres_et_al.pdf. [Consultado el: 20 de diciembre de 2009].

García de Torres, E.; Martínez Martínez, S.; Cebrián, B.; Rodríguez, J. (2006): “La noticia de portada en la prensa digital española y mexicana”, en Sabéz, F. (Ed.): Análisis y propuestas en torno al periodismo digital. Actas del VII Congreso Nacional de Periodismo digital, marzo de 2006. Huesca: Asociación de la Prensa de Aragón. Disponible en: http://septimo.congresoperiodismo.com. [Consultado el: 20 de diciembre de 2009].

García de Torres, E.; Saiz, J.; Cebrián, B.; Berruti, N. y Lavilla, M.J. (2004a). "Prensa digital española en primera línea”, 70 años de periodismo y comunicación en primera línea, VII Congreso Latinoamericano de Investigadores de la Comunicación. Buenos Aires, 11 al 16 de octubre de 20004. Gobierno de la Provincia y Secyt.

- (2004b): "Propiedad, difusión y potencial interactivo y multimedia de los diarios españoles en Internet", Revista Comunicación y Estudios Universitarios, 12, pp. 93-106.

- (2004c): “The portal strategy. A study of Spanish online newspapers' added value”, en Salaverría, R. y Sádaba, C. (Coords.): Towards new media paradigms. Content, Producers, Organisations and Audiences. II Cost A20 International Conference Proceedings, 27-28 de June 2003. Pamplona: Eunate.

Greer, J. y Mensing, D. (2006): “The Evolution of Online Newspapers: A longitudinal content analysis, 1997-2003”, en Li, X. (Ed.): Internet Newspapers: The Making of a Mainstream Medium (pp.13-32). Londres: Routlegde. 
Interactive Advertising Bureau. (2010). El Panorama Interactivo de los grupos de prensa y revistas en España. Cuadernos de comunicación Interactiva. El Libro Blanco vol. 10 de IAB. Revista de la comunicación interactiva y el marketing digital. IAB. Disponible en: http://www.iabspain.net/ver.php?mod=descargas\&id_categoria=9. [Consultado el 26 de julio de 2011].

López Carreño, R. (2004): “Análisis taxonómico de los portales periodísticos españoles”, Anales de Documentación, 2004, n7, pp. 123-140.

Martínez Martínez, S. (2007): “Prensa especializada en Internet: la interactividad y plataformas de distribución”, en Sabés, F. y Verón, J.J (Coord.): Presente y futuro de la Comunicación Digital. Actas del VIII Congreso Nacional de Periodismo Digital, marzo de 2007. Huesca: Asociación de la Prensa de Aragón, pp. 247-262. Disponible en: http://octavo. congresoperiodismo.com. [Consultado el: 28 de marzo de 2009].

- (2010): La interactividad y las plataformas de distribución de los medios online de referencia. Tendencias en la configuración del nuevo entorno informativo digital español. Tesis doctoral dirigida por la Dra. Elvira García de Torres. Defendida el 4 de abril de 2011. Valencia: Universidad CEU Cardenal Herrera.

Martínez Méndez, F. J.; López Carreño, R. (2005): "Análisis prospectivo de las tendencias de desarrollo de los portales periodísticos españoles", Scire, septiembre-diciembre 2005, 11:2, pp. 33- 62. Disponible en: http://ibersid.eu/ ojs/index.php/scire/article/view/1520/1498. [Consultado el 19 de julio de 2011].

Noticias de la Comunicación (2008): "Magnitudes de los principales grupos españoles de prensa diaria”, Noticias de la Comunicación, 285, noviembre de 2008, pp. 52-54.

- (2007): “Magnitudes de los principales grupos españoles de prensa diaria”, Noticias de la Comunicación, 274, noviembre de 2007, pp. 56-58

OJD (2006a): “Medios impresos. Diarios- datos 2005”, ojd.es. Disponible en: http://www.ojd.es/ojdx4/diarios2.asp. [Consultado el: 30 de mayo de 2006]

- (2006b): "PGD. Diarios de Distribución gratuita- datos 2005”, ojd.es. Disponible en: http://www.ojd.es/PGD/ Portal/home_pgd/_FoY9_fRQEwywGyQFe-tmuw. [Consultado el: 30 de mayo de 2006].

- (2006c): “OJDinteractiva. Diarios. Mes abril 2006”, ojd.es. Disponible en: http://www.ojdinteractiva.es/. [Consultado: el 30 de mayo de 2006].

- (2007a): “Medios impresos. Diarios- datos 2006”, ojd.es. Disponible en: http://www.ojd.es/ojdx4/diarios2.asp. Consultado: el 30 de mayo de 2007.

- (2007b): "PGD. Diarios de Distribución gratuita- datos 2006", ojd.es. Disponible en: http://www.ojd.es/PGD/ Portal/home_pgd/_FoY9_fRQEwywGyQFe-tmuw. [Consultado: el 30 de mayo de 2007].

- (2007c): “OJDinteractiva. Diarios. Mes abril 2007”, ojd.es. Disponible en: http://www.ojdinteractiva.es/. [Consultado: el 30 de mayo de 2007]. 
- (2008a): "Medios impresos. Diarios- datos 2007", ojd.es. Disponible en: http://www.ojd.es/ojdx4/diarios2.asp. [Consultado: el 30 de mayo de 2008].

- (2008b): "PGD. Diarios de Distribución gratuita- datos 2007", ojd.es. Disponible en: http://www.ojd.es/PGD/ Portal/home_pgd/_FoY9_fRQEwywGyQFe-tmuw. [Consultado: el 30 de mayo de 2008].

- (2008c): “OJDinteractiva. Diarios. Mes abril 2008”, ojd.es. Disponible en: http://www.ojdinteractiva.es/. [Consultado: el 30 de mayo de 2008].

Ramos Simón, L. F. (1996): “Concentraciones y competencia en los grupos de comunicación multimedia”, Cuadernos de documentación multimedia, no5. Disponible en: http://www.ucm.es/info/multidoc/multidoc/revista/cuadern5/ ramos.htm. [Consultado el: 20 de julio de 2011].

Rost, A. (2006): La interactividad en el periódico digital. Tesis doctoral dirigida por el Dr. Miquel Rodrigo Alsina. Bellaterra: Depósito Biblioteca Comunicación, Universitat Autònoma de Barcelona.

Salaverría, R. (2010):"Estructura de la Convergencia", en López García, X.; Pereira Fariña, X. (Coords.): Convergencia Digital. Reconfiguración de los Medios de Comunicación en España. Santiago de Compostela: Universidade de Santiago de Compostela, pp. 27-40.

- (2003): “Ensayo. Convergencia de medios", Chasqui, 81. Disponible en: http://chasqui.comunica.org/81/salaverria81.htm. [Consultado el: 25 de mayo de 2009]

Santamaría, J.V. (2011): “Reorganización en los grupos multimedia españoles: la nueva cartografía”, Observatorio (OBS) Journal, vol. 5, no 1, pp. 157-174. http://obs.obercom.pt/index.php/obs/article/viewArticle/376. Consultado el: agosto de 2011.

Schultz, T. (1999): "Interactive Options in Online Journalism: a Content Analysis of 100 U.S. Newspapers", Journal of Computer Mediated Communication, 5 (1). Disponible en: http://jcmc.indiana.edu/vol5/issuel/schultz.html. [Consultado el: 25 de mayo de 2011].

The Bivings Group (2007): “American Newspaper and the Internet: Threat or Opportunity?”, bivingsreport.com. Disponible en: http://www.bivingsreport.com/2007/american-newspapers-and-the-internet-threat-or-opportunity/. [Consultado el: 25 de Julio de 2011]. 